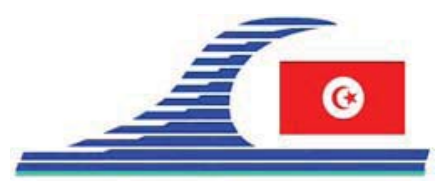

\author{
Conférence Méditerranéenne Côtière et Maritime \\ EDITION 1, HAMMAMET, TUNISIE (2009) \\ Coastal and Maritime Mediterranean Conference \\ Disponible en ligne - http://www.paralia.fr - Available online
}

\title{
Introduction à une étude comparée du fonctionnement de la dynamique du trait de côte du littoral algérien occidental et des côtes picardes et normandes françaises : mécanismes et enjeux
}

\section{Laurent DELABY ${ }^{1}$, Djilali BOURAS ${ }^{2}$, Salim MOUFFOK ${ }^{2}$}

1. DLIC - Delaby Laurent Ingénieurs Conseils, delabylaurent@yahoo.fr

2. Université d'Oran, Algérie,djilaloran@yahoo.fr

Mots clés : Trait de côte - Dynamique - Recul - Littoral - Dégradation

\section{Introduction}

Nous proposons dans ce papier de poser les bases d'une étude comparative des dynamiques d'évolution des côtes picardes françaises sur une mer à forte marée, la Manche, et des côtes oranaise sur une mer à faible marée, la Méditerranée.

Cette étude est une introduction à un dialogue franco-algérien qui doit porter ses fruits sur plusieurs années. Elle présente une innovation : une collaboration entre une jeune entreprise française (DLIC) et une Université algérienne, l'Université d'Oran. Elle se veut un encouragement au développement de ces partenariats entreprise-recherche.

La compréhension des mécanismes régissant les dynamiques d'évolution des littoraux a une importance déterminante pour les populations côtières qui subissent souvent les effets négatifs des variations du trait de côte : soit que la mer emporte les constructions des côtes à retrait, soit que la mer s'éloigne de constructions maritimes telles que les ports. Ces évolutions demandent donc aux populations côtières de constantes adaptations qui se font dans le cadre de ce que l'on appelle aujourd'hui « la gestion intégrée des zones côtières ». L'étude comparée de ces phénomènes sur une mer à forte marée sur les côtes normandes et picardes et sur une mer à faible marée des côtes oranaises permettra d'identifier les mécanismes propres aux marées, et nous conduira à reconnaître les paramètres communs des effets des variations eustatiques du niveau de la mer liés aux changements climatiques. Nous envisageons de comparer les effets climatiques sur les éboulements de falaises, la Picardie étant soumise à un régime pluvial océanique connaissant régulièrement des gelées (cryoclastie) avec des précipitations réparties régulièrement sur l'année, et le littoral oranais connaissant plutôt un régime de précipitation bi-modal avec des températures hivernales de 1 'ordre de $8^{\circ} \mathrm{C}$ (BOURRAS et al., 2007). Ces différences climatiques entrainent des différences dans les importances relatives de divers phénomènes: haloclastie, hydroclastie, nappes phréatiques, ....

Le travail commun de deux équipes dans deux pays différents conduira à un enrichissement mutuel concernant les méthodes d'études, de recueil et d'archivage des informations, d'adaptation des sociétés à l'environnement changeant des littoraux. 


\section{Présentation du littoral oranais}

Le littoral meuble oranais a largement reculé en raison des aménagements effectués (Macta, Kristel, Ain El Turk, ...), et qui ont affaiblit voire brisé l'apport en alluvions des différents cours d'eaux, en particulier au niveau de la région de la Macta. Par ailleurs, les besoins augmentés en sable et en gravier pour les nombreux projets d'aménagements, de l'industrie et les différentes constructions entraînent une surexploitation des réserves des lits des cours d'eau et celle du domaine côtier (cas de Terga). De même des aménagements tels que les tracés de routes, des projets d'aménagements et construction des agglomérations ont aggravé les glissements, (Canastel, Kristel, Terga). Ainsi, sur la falaise de Canastel, toutes les conditions sont rassemblées pour que de graves déplacements de terrain puissent avoir lieu à n'importe quel moment. De plus de nombreux cours d'eaux ont été déviés de leur trajectoire initiale (Macta, Arzew, Kristel, Oued El Halouf), provoquant une augmentation de la salinité des eaux. Signalons également l'intensité des travaux de construction sur des espaces non constructibles (Oran, Cap Falcon, ...), engendrant un fort danger sur l'homme et une déstabilisation et dégradation des reliefs et de l'écosystème. Ceci, impose la nécessité de retracer la dynamique du trait de côte pour une durée bien déterminée. L'empilement événementiel diversifié, de faible fréquence, (érosion, éboulement, glissement), dont l'origine marine ou continentale collabore dans la dynamique et l'évolution du littoral.

Les falaises littorales sont sous l'influence de plusieurs facteurs dont l'action des vagues; l'évolution des versants comme les déplacements en masse; les transformations subaériennes de la météorisation.

Les vagues creusent et érodent la base de la falaise. Parmi les éléments décideurs dans la morphologie d'une falaise, on note la nature des corps constituants : les falaises les plus résistantes et abruptes sont formées de matériels durs (falaises, corniche oranaise, Kristel), alors que celles tendres ont une composition fragile (falaises sablo-gréseuses de Terga et Oued El Halouf). Ces dernières sont souvent soumises à des affaissements, des éboulements et des glissements de terrain. Notons également le pendage des couches qui joue un rôle érosif très important. Quand une falaise régresse, elle laisse un platier rocheux, charpenté par les vagues, comme à Marsa El Hadjadj. La forme des falaises est essentiellement fonction de la nature et de la structure rocheuse. Ce dernier point joue un rôle important par la variabilité de la résistance de la roche et, par conséquent, on aura des tranches de la falaise isolée. Donc le recul de la falaise et le développement de la plate-forme forment des isolements rocheux abrupts appelés généralement aiguilles comme le cas de la Pointe de l'Aiguille (Kristel).

\section{Présentation des côtes normandes et picardes}

Les littoraux étudiés se situent entre Antifer en Normandie et Ault en Picardie, et plus particulièrement sur une bande de $7 \mathrm{~km}$ entre Mers-Les-Bains et Ault. Ces littoraux sont 
principalement formés de falaises de craie tendres du crétacé entre le Turonien supérieur au niveau de la plate forme d'abrasion et le Coniacien pour les parties les plus récentes, cette craie contient entre 2 et $5 \%$ de silex qui forment ensuite les plages de galets protégeant les falaises de l'érosion.
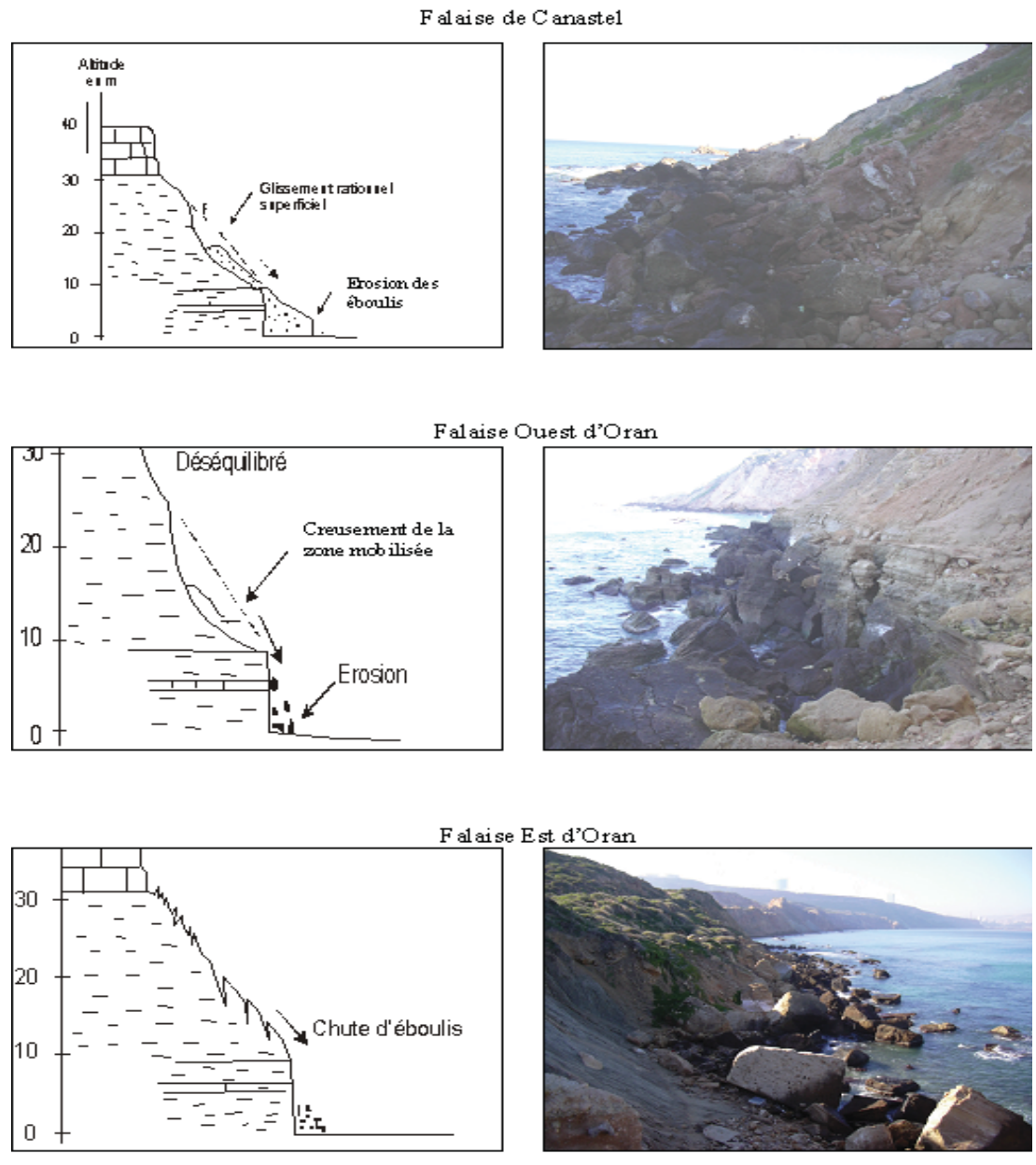

Figure - Différentes formes de dégradation de côte à falaise sur le littoral oranais.

Ces littoraux subissent un recul important liés d'une part à l'élévation du niveau de la mer depuis plusieurs siècles, et d'autres part aux actions anthropiques de prélèvements de galets pour des fins entre autres de constructions, des ports et de jetées qui entravent le transit sédimentaires. Ce recul a été estimé de l'ordre de $30 \mathrm{~cm}$ par an entre Ault et Mers-Les-Bains (DOLIQUE, 1991). Ce recul s'inscrit dans la durée, en 1837 Victor Hugo écrivait déjà : "La mer ronge le bourg d'Ault. Il y a cinquante ans, c'était un bien plus grand village qui avait sa partie basse abritée par la falaise au bord de la mer. Mais un jour la colonne des flots qui descend de la Manche s'est appuyée si violemment sur cette falaise qu'elle l'a fait ployer. La falaise s'est rompue et le village a été englouti. Il n'était resté debout dans l'inondation qu'une ancienne halle et une vieille église ...". La solution idéale pour les populations littorales n'a pas encore été trouvée : si les digues de protection permettent en certains points de ralentir le recul, elles 
engendrent aussi des conséquences collatérales en diminuant l'apport de galets sur les plages, donc une érosion accélérées sur d'autres points du rivage. Elles nécessitent en outre des efforts d'entretien important après leur construction, efforts devant être maintenus constant au travers du temps, et donc une grande capacité des différents acteurs à travailler ensemble.

Un facteur d'influence propre aux côtes à forte marées : les éboulements se gorgent d'eau à marée haute, et se "vident» à marée basse par d'importants écoulements qui contribuent à séparer les constituants en transportant de grandes quantités de sable. Sur la figure précédente, l'éboulement a pratiquement disparu deux ans après sa formation.

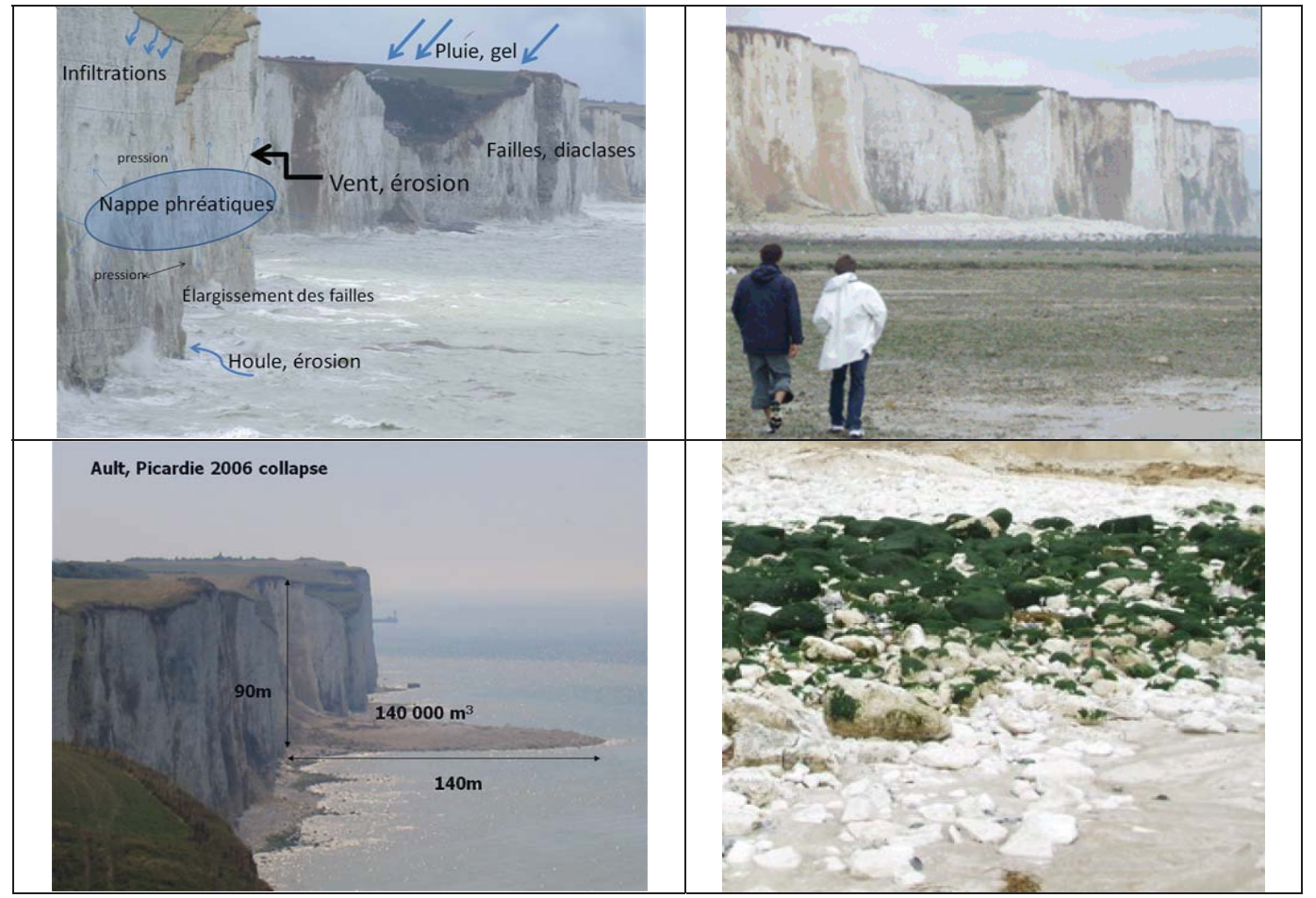

\section{Conclusion}

Ce papier est une introduction, la première pierre d'un long dialogue et d'un long chemin, d'échanges fructueux et de découverte conjointe de littoraux différents, sur des mers différentes, qui abritent des populations et des cultures différentes.

\section{Références bibliographiques}

DOLIQUE F. (1991). L'évolution du littoral entre Dieppe et Le Hourdel (1939-1989). Mémoire de maîtrise, Université de Picardie Jules Verne, Amiens.

BOURAS D., MOUFFOK S., MAATALAH A., BOUTIBA Z. (2007). Dynamique bioclimatique du littoral occidental algérien. LARHYSS Journal, $\mathrm{N}^{\circ}$ 6, pp 45-50. HUGO V. (1837). Extraits de : BLIN, Jean-Pierre, BALANDRA, Eric. Victor Hugo. Lettres et dessins de Picardie. Amiens : Association Monuments de Picardie, 1985. 